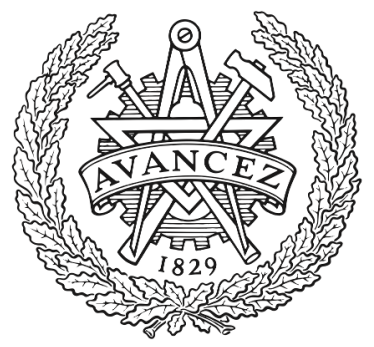

CHALMERS

UNIVERSITY OF TECHNOLOGY

\title{
The State-of-the-art Research on UWB Eleven Feed and QRFH Feed in a Perspective of SKA
}

Downloaded from: https://research.chalmers.se, 2023-04-26 00:15 UTC

Citation for the original published paper (version of record):

Yang, J. (2017). The State-of-the-art Research on UWB Eleven Feed and QRFH Feed in a Perspective of SKA. 2017 IEEE RADIO AND ANTENNA DAYS OF THE INDIAN OCEAN (RADIO). http://dx.doi.org/10.23919/RADIO.2017.8242258

N.B. When citing this work, cite the original published paper. 


\title{
The State-of-the-art Research on UWB Eleven Feed and QRFH Feed in a Perspective of SKA
}

\author{
Jian Yang \\ Dept. of electrical engineering \\ Chalmers University of Technology \\ Gothenburg, Sweden \\ jian.yang@chalmers.se
}

\begin{abstract}
Several large radio astronomy projects, such as SKA, FAST and VLBI, are under development in different phases towards their final goals. Among all future radio telescopes, ultra-wideband (UWB) antenna systems are demanded and thus draws a lot of research resources. In this talk, the state-of-the-art research at Chalmers University of Technology and Onsala Space Observatory on two most promising UWB feed technologies, the Eleven feed and quadruple-ridge flared horn (QRFH) feed, will be reviewed with theory of their working principles, new techniques applied for improving the performance, optimization technologies, mechanical designs for assurance of realization of all technique implementation, and system performance optimization and evaluations. The developments have been driven in a perspective of SKA Mid band array. Examples and future developments will be discussed in the talk.
\end{abstract}

\section{INTRODUCTION}

Among large radio astronomy projects, such as SKA, VLBI and FAST, ultra-wideband (UWB) radio telescopes are critical essential parts for achieving the ultra-sensitivity goals set for these next generation projects. Therefore, ultra-wideband feed technologies have been driven by these high demands to gain rapid developments recent years.

Two major technologies of the UWB feeds are the Eleven feed technology [1], [2] and the Quadruple-ridge Flared Horn (QRFH) feed technology, both with high aperture efficiency and low ohmic loss and different pros and cons so it is important to choose a proper one for different applications.

An overview of the developments of the two technologies, with new results and discussions of the future developments will be presented in the talk.

\section{ELEVEN FEED}

The basic configuration of the Eleven feed is a pair of dipole over a ground plane. For UWB performance, a pair of the cascaded folded dipole array is employed for one polarization so there are two such pairs of arrays for dual polarization. Fig. 1 shows different versions of the Eleven feeds. The main advantages of the Eleven feed compared to the alternative UWB feeds are the nearly constant beamwidth and the fixed phase center location. Furthermore, the Eleven feed is one of the most compact decade-bandwidth feeds for reflectors.

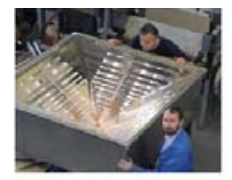

$0.15-2 \mathrm{GHz}$

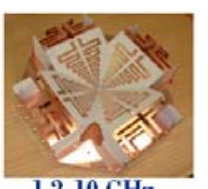

$1.2-10 \mathrm{GHz}$

SKA

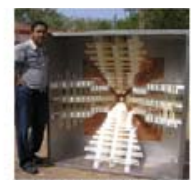

$0.2-0.8 \mathrm{GHz}$ India GMRT

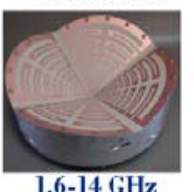

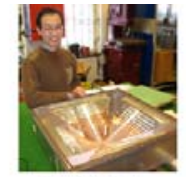

$0.5-3 \mathrm{GHz}$ Russia Ratan

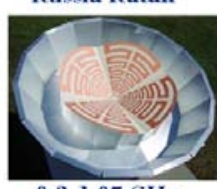

$0.3-1.05 \mathrm{GHz}$

SKA

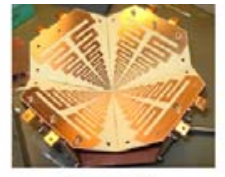

$2-13 \mathrm{GHz}$ VLBI2010

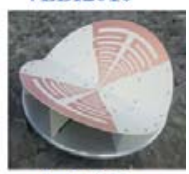

4-18 GHz

feed
Fig. 1 Different versions of the Eleven feeds

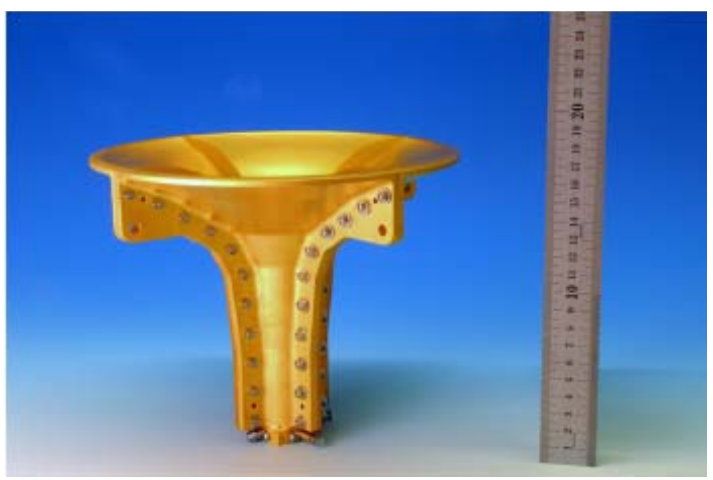

Fig. 2 Prototype of QRFH feed for SKA band B

\section{QRFH FEED}

A QRFH has its own advantages: the standard 50- $\Omega$ coax output ports, all metal structure and the freedom of controlling the beamwidth via changing its profiles. In this talk I will present a new optimization and realization of a quadrupleridge flared horn with new spline-defined profiles instead of the conventional analytical profiles in order to achieve high aperture efficiency and sensitivity over a 5:1 bandwidth at high working frequency up to $24 \mathrm{GHz}$. The spline-profiled QRFH provides a better control of the propagation modes in the horn, which leads to a better aperture efficiency in terms of 
polarization efficiency $\epsilon$ pol, BOR1 efficiency $\epsilon_{\mathrm{BOR} 1}$ and spillover efficiency $\epsilon_{\text {sp. }}$ Optimization technology and mechanical realization for this QRFH feed will be presented in the talk.

\section{REFERENCES}

[1] J. Yang, M. Pantaleev, P.-S. Kildal, B. Klein, Y. Karandikar, L. Helldner, N. Wadefalk,C. Beaudoin, "Cryogenic 2-13 GHz Eleven feed for reflector antennas in future wideband radio telescopes", IEEE Trans. on Antennas Propag., vol. 59, no. 6, pp. 1918-1934, June 2011.

[2] J Yang, M. Pantaleev, B. Billade, M. Ivashina, T. Carozzi, L. Helldner, Leif and M. Dahlgren, "A Compact Dual-Polarized 4-Port Eleven Feed
With High Sensitivity for Reflectors Over 0.35-1.05 GHz", IEEE Trans. Antennas Propag., vol. 63, no. 12, pp. 5955-5960, 2015.

[3] A. Akgiray, S. Weinreb, W. A. Imbriale, and C. Beaudoin, "Circular quadruple-ridged flared horn achieving near-constant beamwidth over multioctave bandwidth: Design and measurements," IEEE Trans. Antennas Propag., vol. 61, no. 3, pp. 1099-1108, 2013.

[4] J. Yang, J. Flygare, M. Pantaleev, and B. Billade, "Development of quadruple-ridge flared horn with spline-defined profile for band B of the Wide Band Single Pixel Feed (WBSPF) advanced instrumentation programme for SKA," in IEEE Int. Symp. Antennas Propag., June 2016, pp. $1345-1346$. 